\title{
A new four-parameter lifetime distribution
}

DOI:

10.1080/02664763.2016.1182137

\section{Document Version}

Accepted author manuscript

Link to publication record in Manchester Research Explorer

\section{Citation for published version (APA):}

Alizadeh, M., Fazel Bagheri, S., Alizadeh, M., \& Nadarajah, S. (2016). A new four-parameter lifetime distribution. Journal of Applied Statistics, 1-31. https://doi.org/10.1080/02664763.2016.1182137

\section{Published in:}

Journal of Applied Statistics

\section{Citing this paper}

Please note that where the full-text provided on Manchester Research Explorer is the Author Accepted Manuscript or Proof version this may differ from the final Published version. If citing, it is advised that you check and use the publisher's definitive version.

\section{General rights}

Copyright and moral rights for the publications made accessible in the Research Explorer are retained by the authors and/or other copyright owners and it is a condition of accessing publications that users recognise and abide by the legal requirements associated with these rights.

\section{Takedown policy}

If you believe that this document breaches copyright please refer to the University of Manchester's Takedown Procedures [http://man.ac.uk/04Y6Bo] or contact uml.scholarlycommunications@manchester.ac.uk providing relevant details, so we can investigate your claim.

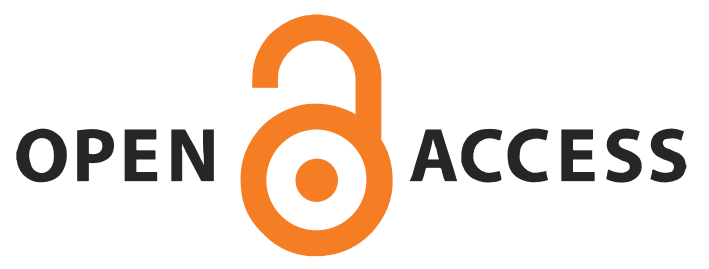




\title{
A new four-parameter lifetime distribution
}

\author{
by \\ Mojtaba Alizadeh \\ Branch of Mazandaran, Statistical Center of Iran \\ Seyyed Fazel Bagheri \\ Department of Mathematics, Islamic Azad University, Tehran, Iran \\ Mohammad Alizadeh \\ Department of Mathematical Sciences, University of Mazandaran, Mazandaran, Iran \\ Saralees Nadarajah \\ School of Mathematics, University of Manchester, Manchester M13 9PL, UK
}

\begin{abstract}
Generalizing lifetime distributions is always precious for applied statisticians. In this paper, we introduce a new four-parameter generalization of the exponentiated power Lindley (EPL) distribution, called the exponentiated power Lindley geometric (EPLG) distribution, obtained by compounding EPL and geometric distributions. The new distribution arises in a latent complementary risks scenario, in which the lifetime associated with a particular risk is not observable; rather, we observe only the maximum lifetime value among all risks. The distribution exhibits decreasing, increasing, unimodal and bathtub-shaped hazard rate functions, depending on its parameters. It contains several lifetime distributions as particular cases: exponentiated power Lindley (EPL), new generalized Lindley (NGL), generalized Lindley (GL), power Lindley (PL) and Lindley geometric (LG) distributions. We derive several properties of the new distribution such as closed-form expressions for the density, cumulative distribution function, survival function, hazard rate function, the $r$ th raw moment, and also the moments of order statistics. Moreover, we discuss maximum likelihood estimation and provide formulas for the elements of the Fisher information matrix. Simulation studies are also provided. Finally, two real data applications are given for showing the flexibility and potentiality of the new distribution.
\end{abstract}

Keywords: Exponentiated power Lindley distribution, Maximum likelihood estimation, Model selection criteria, Probability weighted moments, Residual life function.

\section{Introduction}

In many practical situations, classical probability distributions do not provide adequate fits to real data. For example, if the data are asymmetric, the normal distribution will not be a good choice. So, several methods for generating new probability distributions by adding one or more parameters have been studied in the statistical literature recently. Among these methods, the compounding of some discrete and important lifetime distributions has been in the vanguard of lifetime modelling. Adamidis and Loukas (1998) pioneered a two-parameter exponential-geometric (EG) distribution by compounding the exponential and geometric distributions. In a similar manner, the following distributions were proposed: exponential-logarithmic (EL) distribution (Tahmasbi and Rezaei, 2008); exponential-power series (EPS) distribution (Chahkandi and Ganjali, 2009); Weibull-geometric (WG) distribution (Barreto-Souza et al., 2011); Weibull-power series (WPS) distribution (Morais and Barreto-Souza, 2011); exponentiated exponential-Poisson (EEP) distribution (Barreto-Souza 
and Cribari-Neto, 2009); complementary exponential-geometric (CEG) distribution (Louzada-Neto et al., 2011); complementary Weibull geometric distribution (Tojeiro et al., 2014); Burr XII negative binomial distribution (Ramos, 2015); compound class of extended Weibull power series distributions (Silva, 2013); compound class of linear failure rate-power series distributions (Mahmoudi and Jafari, 2014); exponentiated Weibull-logarithmic distribution (Mahmoudi and Sepahdar, 2013); exponentiated Weibull-logarithmic distribution (Mahmoudi et al., 2014); generalized exponential power series distribution (Mahmoudi and Jafari, 2012); exponentiated Weibull power series distribution (Mahmoudi and Shiran, 2012); generalized modified Weibull power series distribution (Bagheri et al., 2015).

Lindley (1958) introduced a one-parameter distribution, known as Lindley distribution, given by its probability density function (pdf)

$$
f_{L}(x)=\frac{\lambda^{2}}{\lambda+1}(1+x) e^{-\lambda x}
$$

where $x>0$ and $\lambda>0$. Ghitany et al. (2008) discussed various properties of this distribution and showed that in many ways the pdf (1) provides a better model for some applications than the exponential distribution. Bakouch et al. (2012) obtained an extended Lindley distribution and discussed its various properties and applications. Ghitany et al. (2011) developed a twoparameter weighted Lindley distribution and discussed its applications to survival data. Nadarajah et al. (2011) obtained a generalized Lindley distribution and discussed various properties and applications. Merovci and Elbatal (2014) used the quadratic rank transmutation map in order to generate a flexible family of probability distributions taking Lindley-geometric distribution as the base value distribution by introducing a new parameter that would offer more distributional flexibility. Asgharzadeh et al. (2014) introduced a general family of continuous lifetime distributions by compounding any continuous distribution and the Poisson-Lindley distribution. Oluyede and Yang (2014) proposed a new four-parameter generalized Lindley (GLD) distribution called the beta-generalized Lindley distribution (BGLD). This distribution contains the beta-Lindley, GLD and Lindley distributions as particular cases. A two-parameter power Lindley distribution (PLD) has been suggested by Ghitany et al. (2013). A new class of generalized power Lindley distributions with applications to lifetime data has been proposed by Pararai et al. (2015).

The aim of this paper is to propose a new class of lifetime distributions called the exponentiated power Lindley geometric (EPLG) distribution. The distributional properties including survival function, hazard rate function, reverse hazard rate function, limiting behavior of hazard rate function, quantile function, moments, distribution of order statistics, mean deviations, Lorenz and Bonferroni curves and Fisher information are presented. The method of maximum likelihood estimation (MLE) is used to estimate parameters of this new class of distributions. This distribution contains several lifetime distributions as particular cases: exponentiated power Lindley (EPL), new generalized Lindley (NGL), generalized Lindley (GL), power Lindley (PL) and Lindley geometric (LG) distributions. Simulation study is presented to assess the performance and accuracy of the MLEs. Two real data examples are discussed to illustrate the usefulness and applicability of the EPLG distribution.

The contents of this paper are organized as follows. In Section 2, we define the EPLG distribution. The pdf, survival function and hazard rate function and some of their properties are given in this section. We derive quantiles, moments, moment generating function, moments of order statistics of the EPLG distribution in Section 3. In Section 4, we discuss probability weighted moments, residual and reverse residual life functions, mean deviations, Bonferroni and Lorenz curves, reliability of the EPLG distribution. Some particular cases of the EPLG distribution are discussed 
in Section 5. In Section 6, estimation of the parameters by maximum likelihood and inference for large samples are presented. We present a simulation study in Section 7. In Section 8, applications to two real data sets are given. Finally, conclusions are provided in Section 9.

\section{The EPLG distribution}

The three-parameter exponentiated power Lindley (EPL) distribution was introduced by Ashour and Eltehiwy (2014). The cumulative distribution function (cdf) of the EPL distribution with parameters $\alpha, \beta, \lambda>0$ is

$$
G(x)=\left[1-\left(1+\frac{\lambda x^{\beta}}{\lambda+1}\right) e^{-\lambda x^{\beta}}\right]^{\alpha}
$$

The pdf of the EPL distribution is

$$
g(x)=\frac{\alpha \beta \lambda^{2} x^{\beta-1}}{\lambda+1}\left(1+x^{\beta}\right) e^{-\lambda x^{\beta}}\left[1-\left(1+\frac{\lambda x^{\beta}}{\lambda+1}\right) e^{-\lambda x^{\beta}}\right]^{\alpha-1} .
$$

Let $X_{1}, \ldots, X_{N}$ be independent and identical EPL random variables with cdf and pdf given by (2) and (3). Let $N$ be a geometric random variable independent of $X_{1}, \ldots, X_{N}$ with probability mass function

$$
P(N=n)=(1-\theta) \theta^{n-1}, \quad n=1,2, \ldots, \quad \theta>0 .
$$

Also let $X=\max \left(X_{1}, \ldots, X_{N}\right)$, then the cdf of $X$ given $N=n$ is

$$
F_{X \mid N=n}(x)=\left[1-\left(1+\frac{\lambda x^{\beta}}{\lambda+1}\right) e^{-\lambda x^{\beta}}\right]^{\alpha n}
$$

which is an EPL cdf with parameters $\alpha n, \beta$, and $\lambda$. The EPLG distribution, denoted by EPLG $(\alpha, \beta, \lambda, \theta)$, is defined by the marginal cdf of $X$, i.e.

$$
F_{E P L G}(x)=\frac{(1-\theta)\left[1-\left(1+\frac{\lambda x^{\beta}}{\lambda+1}\right) e^{-\lambda x^{\beta}}\right]^{\alpha}}{\left(1-\theta\left[1-\left(1+\frac{\lambda x^{\beta}}{\lambda+1}\right) e^{-\lambda x^{\beta}}\right]^{\alpha}\right)},
$$

where $\alpha, \beta, \lambda, \theta>0$. The pdf of EPLG $(\alpha, \beta, \lambda, \theta)$ is

$$
f_{E P L G}(x)=\frac{\frac{\alpha \beta(1-\theta) \lambda^{2} x^{\beta-1}}{\lambda+1}\left(1+x^{\beta}\right) e^{-\lambda x^{\beta}}\left[1-\left(1+\frac{\lambda x^{\beta}}{\lambda+1}\right) e^{-\lambda x^{\beta}}\right]^{\alpha-1}}{\left(1-\theta\left[1-\left(1+\frac{\lambda x^{\beta}}{\lambda+1}\right) e^{-\lambda x^{\beta}}\right]^{\alpha}\right)^{2}} .
$$

The survival and hazard rate functions of the EPLG distribution are

$$
S_{E P L G}(x)=1-\frac{(1-\theta)\left[1-\left(1+\frac{\lambda x^{\beta}}{\lambda+1}\right) e^{-\lambda x^{\beta}}\right]^{\alpha}}{\left(1-\theta\left[1-\left(1+\frac{\lambda x^{\beta}}{\lambda+1}\right) e^{-\lambda x^{\beta}}\right]^{\alpha}\right)},
$$

and

$$
h_{E P L G}(x)=\frac{\frac{\alpha \beta(1-\theta) \lambda^{2} x^{\beta-1}}{\lambda+1}\left(1+x^{\beta}\right) e^{-\lambda x^{\beta}}\left[1-\left(1+\frac{\lambda x^{\beta}}{\lambda+1}\right) e^{-\lambda x^{\beta}}\right]^{\alpha-1}}{\left(1-\left[1-\left(1+\frac{\lambda x^{\beta}}{\lambda+1}\right) e^{-\lambda x^{\beta}}\right]^{\alpha}\right)\left(1-\theta\left[1-\left(1+\frac{\lambda x^{\beta}}{\lambda+1}\right) e^{-\lambda x^{\beta}}\right]^{\alpha}\right)} .
$$


Proposition 2.1. The limiting distribution of $E P L G(\alpha, \beta, \lambda, \theta)$ as $\theta \rightarrow 0^{+}$is

$$
\lim _{\theta \rightarrow 0^{+}} F(x)=\left[1-\left(1+\frac{\lambda x^{\beta}}{\lambda+1}\right) e^{-\lambda x^{\beta}}\right]^{\alpha},
$$

which is the cdf of the EPL distribution.

Proof. The proof is straightforward.

Figure 1: Plots of pdf, cdf and hazard rate function of the EPLG distribution: pdf for $(\alpha, \beta, \lambda)=$ $(0.2,2,0.2)$ (top left) and pdf for $(\alpha, \beta, \lambda)=(0.6,1.5,0.9)$ (top middle), cdf for $(\alpha, \beta, \lambda)=$ $(0.2,2,0.1)$ (top right) and cdf for $(\alpha, \beta, \lambda)=(1.2,0.8,1.5)$ (bottom left), hazard rate function for $(\alpha, \beta, \lambda)=(0.2,2,1.2)$ (bottom middle) and hazard rate function for $(\alpha, \beta, \lambda)=(0.1,1.6,0.8)$ (bottom right).

Proposition 2.2. The limiting behavior of the hazard rate function of the EPLG distribution in (6) is

$$
h(x)= \begin{cases}\infty, & \alpha \leq 1, \beta<1 \\ \frac{(1-\theta) \lambda^{2}}{(\lambda+1)}, & \alpha=\beta=1 \\ 0, & \alpha \geq 1, \beta>1\end{cases}
$$

Proof. The proof is straightforward.

Plots of pdf, cdf and hazard rate function of the EPLG distribution for some values of $\alpha, \beta, \lambda$ and $\theta$ are shown in Figures 1 and 2 .

\section{Quantiles, moments and moments of order statistics of the EPLG distribution}

Some of the most important features and characteristics of a distribution can be studied through its moments and quantiles such as dispersion, skewness and kurtosis. Also, the quantiles of a distribution can be used in data generation.

Figure 2: Plots of pdf, cdf and hazard rate function of the EPLG distribution: pdf for $(\alpha, \beta, \lambda)=$ $(0.4,1.5,0.4)$ (top left) and pdf for $(\alpha, \beta, \lambda)=(0.3,1.5,0.3)$ (top middle), cdf for $(\alpha, \beta, \lambda)=$ $(0.8,1.8,0.9)$ (top right) and pdf for $(\alpha, \beta, \lambda)=(0.2,2,0.9)$ (bottom left), hazard rate function for $(\alpha, \beta, \lambda)=(0.2,2,1.8)$ (bottom middle) and hazard rate function for $(\alpha, \beta, \lambda)=(0.2,1.8,1.5)$ (bottom right).

\subsection{Quantiles}

Hence, we propose algorithms for generating random data from the EPLG distribution. Let $X$ denote a random variable with the pdf (5). The quantile function, say $Q(p)$, defined by $F(Q(p))=$ 
$p$, for $0<p<1$, is the root of

$$
\left(1+\frac{\lambda x^{\beta}}{\lambda+1}\right) e^{-\lambda x^{\beta}}=1-(\underbrace{\frac{p}{1-\theta+p \theta}}_{k_{c}})^{\frac{1}{\alpha}}=1-k_{c}^{\frac{1}{\alpha}} .
$$

Substituting $Z(p)=-1-\lambda-\lambda[Q(p)]^{\beta}$, one can rewrite $(7)$ as

$$
Z(p) e^{Z(p)}=-(1+\lambda)\left(1-k_{c}^{\frac{1}{\alpha}}\right) e^{-1-\lambda} .
$$

So, the solution for $Z(p)$ is

$$
Z(p)=W\left(-(1+\lambda)\left(1-k_{c}^{\frac{1}{\alpha}}\right) e^{-1-\lambda}\right)
$$

where $W(\cdot)$ is the Lambert $W$ function, see Corless et al. (1996) for detailed properties. Inverting (8), one obtains

$$
Q(p)=\left[-1-\frac{1}{\lambda}-\frac{1}{\lambda} W\left(-(1+\lambda) e^{-1-\lambda}\left(1-\left(\frac{p}{1-\theta+p \theta}\right)^{\frac{1}{\alpha}}\right)\right)\right]^{\frac{1}{\beta}}
$$

\subsection{Moments}

Moments are necessary and important in any statistical analysis, especially in practical applications. Ashour and Eltehiwy (2014) showed that if $X \sim E P L(\alpha, \beta, \lambda)$, then the $r$ th moment of $X$, say $E\left(X^{r}\right)$, is

$\nu_{r}[\alpha, \beta, \lambda]=E\left(X^{r}\right)=\frac{\alpha}{\lambda+1} C_{i, k}\left[\frac{\Gamma\left(\frac{r+\beta(k+1)}{\beta}\right)}{\left(\lambda^{\frac{r+k \beta}{\beta}-1}\right)\left((i+1)^{\Gamma\left(\frac{r+\beta(k+1)}{\beta}\right)}\right)}+\frac{\Gamma\left(\frac{r+\beta(k+2)}{\beta}\right)}{\left(\lambda^{\frac{r+k \beta}{\beta}}\right)\left((i+1)^{\Gamma\left(\frac{r+\beta(k+2)}{\beta}\right)}\right)}\right]$,

where $C_{i, k}=\sum_{i=0}^{\infty} \sum_{k=0}^{\infty}\left(\begin{array}{c}\alpha-1 \\ i\end{array}\right)\left(\begin{array}{c}i \\ k\end{array}\right)(-1)^{i}\left(\frac{\theta}{\theta+1}\right)^{k}$. Suppose that $Y_{(n)}=\max \left(Y_{1}, \ldots, Y_{n}\right) \sim \mathrm{EPL}$ $(\alpha n, \beta, \lambda)$, then the $r$ th moment of $\operatorname{EPLG}(\alpha, \beta, \lambda, \theta)$ is given by the following.

Proposition 3.1. The rth moment of EPLG $(\alpha, \beta, \lambda, \theta)$ is

$$
\begin{aligned}
\mu_{r}= & \sum_{n=1}^{\infty} \sum_{i=0}^{\infty} \sum_{k=0}^{\infty}\left(\begin{array}{c}
\alpha n-1 \\
i
\end{array}\right)\left(\begin{array}{c}
i \\
k
\end{array}\right)(-1)^{i}\left(\frac{\theta}{\theta+1}\right)^{k} \frac{\alpha n P(N=n)}{\lambda+1} \\
& \cdot\left[\frac{\Gamma\left(\frac{r+\beta(k+1)}{\beta}\right)}{\left(\lambda^{\frac{r+k \beta}{\beta}-1}\right)\left((i+1)^{\Gamma\left(\frac{r+\beta(k+1)}{\beta}\right)}\right)}+\frac{\Gamma\left(\frac{r+\beta(k+2)}{\beta}\right)}{\left(\lambda^{\frac{r+k \beta}{\beta}}\right)\left((i+1)^{\Gamma\left(\frac{r+\beta(k+2)}{\beta}\right)}\right)}\right] .
\end{aligned}
$$

Proof. See Appendix 1. 
The first and second moments of the EPLG distribution can be computed by substituting $r=1$ and $r=2$ into (9).

From (9), the measures of skewness and kurtosis of the EPLG $(\alpha, \beta, \lambda, \theta)$ can be obtained as skewness $=\frac{\mu_{3}-3 \mu_{1} \mu_{2}+2 \mu_{1}^{3}}{\left(\mu_{2}-\mu_{1}\right)^{\frac{3}{2}}}$ and kurtosis $=\frac{\mu_{4}-4 \mu_{3} \mu_{1}+6 \mu_{1}{ }^{2} \mu_{2}-3 \mu_{1}{ }^{4}}{\left(\mu_{2}-\mu_{1}\right)^{2}}$.

Figure 3 plots the behavior of the Galton' skewness (Johnson et al., 1994) and Moors' kurtosis (Moors, 1988) as functions of $\theta$ for representative values of $\alpha, \beta$ and $\lambda$. We conclude that both Galton' skewness and Moors' kurtosis decrease as $\theta$ increases and when $\alpha, \beta, \lambda$ are not very large.

Figure 3: Plots of Galton' skewness (left) and Moors' kurtosis (right) for the EPLG distribution for different values of $\alpha, \beta$ and $\lambda$.

\subsection{Moment generating function}

Here, we obtain the moment generating function of the EPLG distribution. Using (9) and the expansion of $e^{t x}=\sum_{r=0}^{\infty} \frac{t^{r}}{r !} x^{r}$, it can be shown that

$$
\begin{aligned}
M_{X}(t)= & \sum_{r=0}^{\infty} \sum_{n=1}^{\infty} \sum_{i=0}^{\infty} \sum_{k=0}^{\infty}\left(\begin{array}{c}
\alpha n-1 \\
i
\end{array}\right)\left(\begin{array}{c}
i \\
k
\end{array}\right)(-1)^{i}\left(\frac{\theta}{\theta+1}\right)^{k} \frac{t^{r}}{r !} \frac{\alpha n P(N=n)}{\lambda+1} \\
& \cdot\left[\frac{\Gamma\left(\frac{r+\beta(k+1)}{\beta}\right)}{\left(\lambda^{\frac{r+k \beta}{\beta}-1}\right)\left((i+1)^{\Gamma\left(\frac{r+\beta(k+1)}{\beta}\right)}\right)}+\frac{\Gamma\left(\frac{r+\beta(2)}{\beta}\right)}{\left.\left(\lambda^{\frac{r+k \beta}{\beta}}\right)\left((i+1)^{\Gamma\left(\frac{r+\beta(k+2)}{\beta}\right)}\right)\right] .}\right.
\end{aligned}
$$

\subsection{Moments of order statistics}

Order statistics make their appearance in many areas of statistical theory and practice. Moments of order statistics play an important role in quality control testing and reliability, where a practitioner needs to predict the failure of future items based on the times of a few early failures. These predictors are often based on moments of order statistics. We now derive explicit expressions for the pdf and cdf of the $i$ th order statistic, $Y_{i: n}$, in a random sample of size $n$ from the EPLG distribution. By using (4) and (5), the pdf and the cdf of $Y_{i: n}$ for $i=1, \ldots, n$ can be expressed as follows.

Proposition 3.2. The pdf and the cdf of $Y_{i: n}$ are

$$
\begin{aligned}
f_{i: n}(y)= & \frac{1}{B(i, n-i+1)} \frac{\frac{\alpha \beta(1-\theta) \lambda^{2} y^{\beta-1}}{\lambda+1}\left(1+y^{\beta}\right) e^{-\lambda y^{\beta}}\left[1-\left(1+\frac{\lambda y^{\beta}}{\lambda+1}\right) e^{-\lambda y^{\beta}}\right]^{\alpha-1}}{\left(1-\theta\left[1-\left(1+\frac{\lambda y^{\beta}}{\lambda+1}\right) e^{-\lambda y^{\beta}}\right]^{\alpha}\right)^{2}} \\
& \cdot \sum_{j=0}^{n-i}\left(\begin{array}{c}
n-i \\
j
\end{array}\right)(-1)^{j}\left\{\frac{(1-\theta)\left[1-\left(1+\frac{\lambda y^{\beta}}{\lambda+1}\right) e^{-\lambda y^{\beta}}\right]^{\alpha}}{1-\theta\left[1-\left(1+\frac{\lambda y^{\beta}}{\lambda+1}\right) e^{-\lambda y^{\beta}}\right]^{\alpha}}\right\}^{j+i-1}
\end{aligned}
$$


and

$$
F_{i: n}(y)=\sum_{k=i}^{n} \sum_{j=0}^{n-k}\left(\begin{array}{c}
n-k \\
j
\end{array}\right)\left(\begin{array}{l}
n \\
k
\end{array}\right)(-1)^{j}\left\{\frac{(1-\theta)\left[1-\left(1+\frac{\lambda y^{\beta}}{\lambda+1}\right) e^{-\lambda y^{\beta}}\right]^{\alpha}}{1-\theta\left[1-\left(1+\frac{\lambda y^{\beta}}{\lambda+1}\right) e^{-\lambda y^{\beta}}\right]^{\alpha}}\right\}^{j+k},
$$

respectively.

Proof. See Appendix 2.

So, by using (10), the $i$ th moment of the order statistics $\left(Y_{1: n} \leq Y_{2: n} \cdots \leq Y_{n: n}\right)$ becomes

$$
E\left(Y_{i: n}^{k}\right)=\frac{1}{B(i, n-i+1)} \sum_{j=0}^{n-i}\left(\begin{array}{c}
n-i \\
j
\end{array}\right)(-1)^{j} \int_{0}^{\infty} y^{k} f(y)[F(y)]^{j+i-1} d y
$$

Consequently, for $k=1$, we have

$$
\begin{gathered}
E\left(Y_{1: n}\right)=n \sum_{j=0}^{n-1}\left(\begin{array}{c}
n-1 \\
j
\end{array}\right)(-1)^{j} \int_{0}^{\infty} y f(y)[F(y)]^{j} d y \\
E\left(Y_{2: n}\right)=n(n-1) \sum_{j=0}^{n-2}\left(\begin{array}{c}
n-2 \\
j
\end{array}\right)(-1)^{j} \int_{0}^{\infty} y f(y)[F(y)]^{j+1} d y,
\end{gathered}
$$

and

$$
E\left(Y_{n: n}\right)=n \int_{0}^{\infty} y f(y)[F(y)]^{n-1} d y
$$

\section{Other properties of the EPLG distribution}

Here, we derive some of the other properties of EPLG distribution. For more simple notation, throughout we define $P(N=n)=\frac{\theta^{n}}{T(\theta)}$ in which $T(\theta)=\theta(1-\theta)^{-1}$ and $F(x)=\frac{T(\theta G(x))}{T(\theta)}$.

\subsection{Probability weighted moments}

Probability weighted moments (PWMs) are expectations of certain functions of a random variable defined when the ordinary moments of the random variable exist. The PWMs method can generally be used for estimating parameters of a distribution whose inverse form cannot be expressed explicitly. Estimates based on PWMs are often considered to be superior to standard moment-based estimates. They are sometimes used when MLEs are unavailable or difficult to compute. They may also be used as starting values for MLEs. The PWMs method, which has been investigated by many researchers, was originally proposed by Greenwood et al. (1979). Since then it has been used widely in practice for research purposes. Hosking et al. (1985) investigated the PWMs method for the generalized extreme value (GEV) distribution using fairly long observed series, and they gave a good summary of the PWMs method. Hosking (1986) showed that the PWMs method is superior to the ML method in parameter estimation when the extreme value distribution is used for longer return periods. For a random variable with the pdf $f(\cdot)$ and $\operatorname{cdf} F(\cdot)$, the PWMs are defined by

$$
\Psi_{s, r}=E\left(X^{s} F(X)^{r}\right)=\int_{0}^{\infty} x^{s} F(x)^{r} f(x) d x .
$$

For the EPLG $(\alpha, \beta, \lambda, \theta)$ distribution, the PWMs are given by the following. 
Proposition 4.1. For the EPLG $(\alpha, \beta, \lambda, \theta)$ distribution,

$$
\Psi_{s, r}=A_{2} \sum_{n=0}^{\infty} \frac{b_{n+1}^{*} \theta^{n+1}}{n} \nu_{s}(\alpha n, \beta, \lambda),
$$

where $A_{2}=\left(\frac{1}{T(\theta)}\right)^{r}, b_{0}^{*}=\left(a_{0}^{*}\right)^{n}, a_{n}^{*}=n+1$ and $b_{m}^{*}=\frac{1}{m a_{0}^{*}} \sum_{k=0}^{m}(r k-m+k) a_{k}^{*} b_{m-k}^{*}$. Also, the sth moment of the EPLG distribution can be obtained by setting $r=0$ in $\Psi_{s, r}$.

Proof. See Appendix 3.

\subsection{Residual life function of the EPLG distribution}

Given that a component survives up to time $t \geq 0$, the residual life is the period beyond $t$ until the time of failure and defined by the conditional random variable $X-t \mid X>t$. The mean residual life (MRL) function is an important function in survival analysis, actuarial science, economics and other social sciences and reliability for characterizing lifetime. Although the shape of the hazard rate function plays an important role in repair and replacement strategies, the MRL function is more relevant as the latter summarizes the entire residual life function, whereas the former considers only the risk of instantaneous failure. In reliability, it is well known that the MRL function and ratio of two consecutive moments of residual life determine the distribution uniquely (Gupta and Gupta, 1983). MRL function as well as hazard rate function are very important, since each of them can be used to determine a unique corresponding lifetime distribution. Lifetimes can exhibit IMRL (increasing MRL) or DMRL (decreasing MRL). MRL functions that first decreases (increases) and then increases (decreases) are usually called bathtub-shaped (upside-down bathtub), BMRL (UMRL). The relationship between the behaviors of the two functions of a distribution has been studied by many authors, see Park (1985), Ghitany (1998), Mi (1995) and Tang et al. (1999).

\subsection{The $\tau$ th order moment of the residual life and reversed residual life function}

The following proposition gives a general result on $\tau$ th order moment of the residual life of the EPLG distribution.

Proposition 4.2. The $\tau$ th order moment of the residual life of the $\operatorname{EPL} G(\alpha, \beta, \lambda, \theta)$ distribution is

$$
\begin{aligned}
m_{\tau}(t)= & \frac{1}{S(t)} \sum_{i=0}^{\tau} \sum_{n=1}^{\infty} \sum_{j=0}^{\alpha n-1} \sum_{k=0}^{j}\left(\begin{array}{c}
\tau \\
i
\end{array}\right)\left(\begin{array}{c}
\alpha n-1 \\
j
\end{array}\right)\left(\begin{array}{l}
j \\
k
\end{array}\right)(-t)^{\tau-i} n \frac{\alpha \theta^{n} \lambda^{2}}{\lambda+1}(-1)^{j}\left(\frac{\lambda}{\lambda+1}\right)^{k} \\
& \cdot\left[\Gamma\left(\frac{i}{\beta}+k+1, \lambda(1+j) t^{\beta}\right)+\Gamma\left(\frac{i}{\beta}+k+2, \lambda(1+j) t^{\beta}\right)\right],
\end{aligned}
$$

where $A=1-\left(1+\frac{\lambda x^{\beta}}{\lambda+1}\right) e^{-\lambda x^{\beta}}$.

Proof. See Appendix 4.

The variance of the residual life function of the EPLG distribution can be obtained using $m_{1}(t)$ and $m_{2}(t)$. 
We analogously discuss the reversed residual life and some of its properties. The reversed residual life can be defined as the conditional random variable $t-X \mid X \leq t$ which denotes the time elapsed from the failure of a component given that its life is less than or equal to $t$. This random variable may also be called the inactivity time (or time since failure); for more details see Kundu and Nanda (2010) and Nanda et al. (2003). Also, in reliability, the mean reversed residual life (MRRL) and ratio of two consecutive moments of reversed residual life characterize the distribution uniquely.

Proposition 4.3. The $\tau$ th moment of the reversed residual life function of the EPLG $(\alpha, \beta, \lambda, \theta)$ distribution is

$$
\begin{aligned}
\operatorname{m.reversed}_{\tau}(t)= & \frac{1}{F(t)} \sum_{i=0}^{\tau} \sum_{n=1}^{\infty} \sum_{j=0}^{\alpha n-1} \sum_{k=0}^{j}\left(\begin{array}{l}
\tau \\
i
\end{array}\right)\left(\begin{array}{c}
\alpha n-1 \\
j
\end{array}\right)\left(\begin{array}{l}
j \\
k
\end{array}\right)(-t)^{\tau-i} n \frac{\alpha \theta^{n} \lambda^{2}}{\lambda+1}(-1)^{j}\left(\frac{\lambda}{\lambda+1}\right)^{k} \\
& \cdot\left[\gamma\left(\frac{i}{\beta}+k+1, \lambda(1+j) t^{\beta}\right)+\gamma\left(\frac{i}{\beta}+k+2, \lambda(1+j) t^{\beta}\right)\right],
\end{aligned}
$$

where $A=1-\left(1+\frac{\lambda x^{\beta}}{\lambda+1}\right) e^{-\lambda x^{\beta}}$.

Proof. See Appendix 5.

\subsection{Mean deviations}

The amount of scatter in a population can be measured by the totality of deviations from the mean and median. The mean deviation from the mean is a robust statistic, being more resilient to outliers in a data set than the standard deviation. For a random variable $X$ with pdf $f(x)$, cdf $F(x)$, mean $\mu$ and median $M$, the mean deviation from the mean and the mean deviation from the median are defined by

$$
\delta_{1}(\mu)=\int_{0}^{\infty}|x-\mu| f(x) d x=2 \mu F(\mu)-2 I(\mu)
$$

and

$$
\delta_{2}(M)=\int_{0}^{\infty}|x-M| f(x) d x=2 M F(M)-M+\mu-2 I(M),
$$

respectively, where $I(a)=\int_{0}^{a} x f(x) d x=\varpi_{1}^{t}[\alpha, \beta, \lambda, \theta]=\int_{0}^{t} x f(x) d x$

\subsection{Bonferroni and Lorenz curves}

Study of income inequality has gained a lot of importance over the last few years. Lorenz curve and the associated Gini index are undoubtedly the most popular indices of income inequality. However, there are certain measures which despite possessing interesting characteristics have not been used often for measuring inequality. Bonferroni curve and scaled total time on test transform are two such measures, which have the advantage of being represented graphically in the unit square and also be related to the Lorenz curve and Gini ratio (Giorgi, 1998). These two measures have some applications in reliability and life testing as well (Giorgi and Crescenzi, 2001). The Bonferroni and Lorenz curves and Gini index have many applications not only in economics to study income and 
poverty, but also in other fields like reliability, medicine and insurance. The Bonferroni curve of the EPLG distribution is given by

$$
B_{F}(F(x))=\frac{1}{\mu F(x)} \int_{0}^{x} u f(u) d u=\frac{1}{\mu F(x)} \varpi_{1}^{t}[\alpha, \beta, \lambda, \theta] .
$$

The Lorenz curve of the EPLG distribution can be obtained via

$$
L_{F}(F(x))=B_{F}(F(x)) F(x)=\frac{1}{\mu} \int_{0}^{x} u f(u) d u=\frac{1}{\mu} \varpi_{1}^{t}[\alpha, \beta, \lambda, \theta] .
$$

Some numerical values of $\mu_{1}, \mu_{2}, \mu_{3}, \mu_{4}, M_{X}(t=2)$, skewness, kurtosis, $\delta_{1}(\mu)$ and $\delta_{2}(M)$ of the EPLG distribution for $(\alpha, \beta, \lambda)=(1,1,2),(1,2,1)$ and $\theta=(0.03,0.8,1,1.5,3,3)$ are listed in Table

\begin{tabular}{|c|c|c|c|c|c|}
\hline \multicolumn{6}{|c|}{ The values of the first four moments of the EPLG distribution for $\alpha=1, \beta=1$ and $\lambda=2$} \\
\hline EPLG for $\theta$ : & 0.03 & 0.2 & 0.5 & 0.7 & 0.9 \\
\hline$\mu_{1}$ & 2.248 & 1.297 & 0.911 & 0.787 & 0.701 \\
\hline$\mu_{2}$ & 5.967 & 2.362 & 1.346 & 1.073 & 0.899 \\
\hline$\mu_{3}$ & 17.813 & 5.337 & 2.662 & 2.024 & 1.641 \\
\hline$\mu_{4}$ & 58.830 & 14.308 & 6.549 & 4.848 & 3.856 \\
\hline$M_{X}(t=2)$ & 45577.049 & 2017.731 & 338.521 & 166.394 & 96.282 \\
\hline skewness & 0.005 & 0.071 & 0.153 & 0.191 & 0.221 \\
\hline kurtosis & 0.023 & 0.220 & 0.421 & 0.512 & 0.586 \\
\hline$\delta_{1}(\mu)$ & 0.748 & 0.651 & 0.557 & 0.515 & 0.483 \\
\hline$\delta_{2}(M)$ & 0.748 & 0.645 & 0.543 & 0.497 & 0.462 \\
\hline \multicolumn{6}{|c|}{ The values of the first four moments of the EPLG distribution for $\alpha=1, \beta=2$ and $\lambda=1$} \\
\hline$\theta:$ & 0.03 & 0.8 & 1.5 & 3 & 5 \\
\hline$\mu_{1}$ & 2.136 & 1.595 & 1.316 & 1.214 & 1.139 \\
\hline$\mu_{2}$ & 4.793 & 2.832 & 2.021 & 1.757 & 1.574 \\
\hline$\mu_{3}$ & 11.155 & 5.411 & 3.445 & 2.863 & 2.477 \\
\hline$\mu_{4}$ & 26.772 & 10.957 & 6.364 & 5.109 & 4.305 \\
\hline$M_{X}(t=2)$ & 107.351 & 42.039 & 25.388 & 20.949 & 18.115 \\
\hline skewness & -0.065 & -0.050 & -0.009 & 0.014 & 0.032 \\
\hline kurtosis & -0.188 & -0.121 & -0.004 & 0.052 & 0.097 \\
\hline$\delta_{1}(\mu)$ & 0.367 & 0.427 & 0.434 & 0.430 & 0.425 \\
\hline$\delta_{2}(M)$ & 0.366 & 0.426 & 0.434 & 0.430 & 0.424 \\
\hline
\end{tabular}
1.

Table 1: The values of the first four moments of the EPLG distribution for different parameter values.

\subsection{Reliability}

We derive the reliability $R=P(Y>X)$ when $X \sim \operatorname{EPLG}\left(\alpha_{1}, \beta_{1}, \lambda_{1}, \theta_{1}\right)$ and $Y \sim$ EPLG $\left(\alpha_{2}, \beta_{2}, \lambda_{2}, \theta_{2}\right)$ are independent random variables. It can be shown that

Proposition 4.4. The reliability $R=P(Y>X)$ can be expressed as

$$
\begin{aligned}
R= & \frac{\alpha_{1} \beta_{1} \theta_{1} \lambda_{1}^{2}}{\left(\lambda_{1}+1\right) T\left(\theta_{1}\right) T\left(\theta_{2}\right)} \sum_{n=1}^{\infty} \sum_{i=0}^{\infty} \sum_{n=1}^{\infty} \sum_{j=0}^{\infty}\left(\begin{array}{c}
n \alpha_{1}-1 \\
i
\end{array}\right)(-1)^{i}\left(\begin{array}{c}
n \alpha_{2}-1 \\
j
\end{array}\right)(-1)^{j} n \theta_{1}^{n-1} \theta_{2}^{n} \\
& \cdot \sum_{m=0}^{i} \sum_{l=0}^{j}\left(\begin{array}{c}
i \\
m
\end{array}\right)\left(\begin{array}{l}
j \\
l
\end{array}\right)\left(\frac{\lambda_{1}}{\lambda_{1}+1}\right)^{m}\left(\frac{\lambda_{2}}{\lambda_{2}+1}\right)^{l} .
\end{aligned}
$$

Proof. See Appendix 6. 


\section{Particular cases of the EPLG distribution}

The EPLG distribution contains various particular cases. Some of these distributions are discussed here.

(a) The EPL distribution

The EPL distribution is a particular case of the EPLG distribution for $\theta \longrightarrow 0$ with the cdf and pdf given in (2) and (3). It was introduced by Ashour and Eltehiwy (2014).

(b) The Lindley-geometric (LG) distribution

The LG distribution is a particular case of the EPLG distribution for $\alpha=1$ and $\beta=1$ introduced by Gui et al. (2014). The pdf of the LP distribution is

$$
f_{L G}(x)=\frac{\lambda^{2}}{\lambda+1}(1-\theta)(1+x) e^{-\lambda x}\left[1-\theta\left(1+\frac{\lambda x}{\lambda+1}\right) e^{-\lambda x}\right]^{-2}, 0<\theta<1, x, \lambda>0 .
$$

(c) The power Lindley (PL) distribution

The PL distribution is a particular case of the EPLG distribution for $\theta \longrightarrow 0$ and $\alpha=1$ introduced by Ghitany et al. (2013). The pdf of the PL distribution is

$$
f_{P L}(x)=\frac{\beta \lambda^{2}}{\lambda+1}\left(1+x^{\beta}\right) x^{\beta-1} e^{-\lambda x^{\beta}}
$$

(d) The new generalized Lindley (NGL) distribution

The NGL distribution is a particular case of the EPLG distribution for $\theta \longrightarrow 0$ and $\beta=1$ introduced by Nadarajah et al. (2011). The pdf of the NGL distribution is

$$
f_{N G L}(x)=\frac{\alpha \lambda^{2}}{1+\lambda}(1+x) e^{-\lambda x}\left[1-\left(1+\frac{\lambda x}{\lambda+1}\right) e^{-\lambda x}\right]^{\alpha-1} .
$$

\section{$6 \quad$ Estimation and inference}

Standard statistical techniques such as MLE can always be used for parametric estimation. The likelihood equations, given the complete or censored failure data set, can be derived and solved. Parameter estimation is usually a difficult problem even for the four-parameter EPLG distribution. Methods like the MLE will not yield a closed form solution. Different methods can be used to estimate parameters of the EPLG distribution. Among these methods, the MLE method is the most commonly used method for parameter estimation. Here, we use the ML procedure to derive the point and interval estimates of the parameters. The log-likelihood function is

$$
\begin{aligned}
L= & n \ln (\alpha)+n \ln (\beta)+2 n \ln (\lambda)-n \ln (\lambda+1)+n \ln (1-\theta)+(\beta-1) \sum_{i=1}^{n} \ln \left(x_{i}\right)+\sum_{i=1}^{n} \ln \left(1+x_{i}{ }^{\beta}\right) \\
& -\lambda \sum_{i=1}^{n} x_{i}{ }^{\beta}+(\alpha-1) \sum_{i=1}^{n} \ln \left(1-\left(1+\frac{\lambda x_{i}{ }^{\beta}}{\lambda+1}\right) e^{-\lambda x_{i}{ }^{\beta}}\right) \\
& -2 \sum_{i=1}^{n} \ln \left(1-\theta\left(1-\left(1+\frac{\lambda x_{i}^{\beta}}{\lambda+1}\right) e^{-\lambda x_{i}{ }^{\beta}}\right)^{\alpha}\right) .
\end{aligned}
$$


Calculating the first partial derivatives of $L$ with respect to $\alpha, \beta, \lambda, \theta$ and equating each to zero, we get the derivative of likelihood equations in the following system of nonlinear equations

$$
\begin{aligned}
& \frac{\partial L}{\partial \alpha}=\frac{n}{\alpha}+\sum_{i=1}^{n} \ln \left(1-\left(1+\frac{\lambda x_{i}^{\beta}}{\lambda+1}\right) e^{-\lambda x_{i}{ }^{\beta}}\right) \\
& +2 \sum_{i=1}^{n} \theta\left(1-\left(1+\frac{\lambda x_{i}{ }^{\beta}}{\lambda+1}\right) e^{-\lambda x_{i}{ }^{\beta}}\right)^{\alpha} \ln \left(1-\left(1+\frac{\lambda x_{i}^{\beta}}{\lambda+1}\right) e^{-\lambda x_{i}{ }^{\beta}}\right) \\
& \cdot\left(1-\theta\left(1-\left(1+\frac{\lambda x_{i}^{\beta}}{\lambda+1}\right) e^{-\lambda x_{i}^{\beta}}\right)^{\alpha}\right)^{-1} \text {, } \\
& \frac{\partial L}{\partial \beta}=\frac{n}{\beta}+\sum_{i=1}^{n} \ln \left(x_{i}\right)+\sum_{i=1}^{n} \frac{x_{i}{ }^{\beta} \ln \left(x_{i}\right)}{1+x_{i}{ }^{\beta}}-\lambda \sum_{i=1}^{n} x_{i}{ }^{\beta} \ln \left(x_{i}\right) \\
& +(\alpha-1) \sum_{i=1}^{n}\left(-\frac{\lambda x_{i}{ }^{\beta} \ln \left(x_{i}\right) e^{-\lambda x_{i}{ }^{\beta}}}{\lambda+1}+\left(1+\frac{\lambda x_{i}{ }^{\beta}}{\lambda+1}\right) e^{-\lambda x_{i}{ }^{\beta}} \lambda x_{i}{ }^{\beta} \ln \left(x_{i}\right)\right) \\
& \cdot\left(1-\left(1+\frac{\lambda x_{i}^{\beta}}{\lambda+1}\right) e^{-\lambda x_{i}^{\beta}}\right)^{-1} \\
& +2 \sum_{i=1}^{n} \theta \alpha\left(1-\left(1+\frac{\lambda x_{i}^{\beta}}{\lambda+1}\right) e^{-\lambda x_{i}^{\beta}}\right)^{\alpha} \\
& \cdot\left(-\frac{\lambda x_{i}{ }^{\beta} \ln \left(x_{i}\right) e^{-\lambda x_{i}{ }^{\beta}}}{\lambda+1}+\left(1+\frac{\lambda x_{i}{ }^{\beta}}{\lambda+1}\right) e^{-\lambda x_{i}{ }^{\beta}} \lambda x_{i}{ }^{\beta} \ln \left(x_{i}\right)\right) \\
& \cdot\left(1-\left(1+\frac{\lambda x_{i}^{\beta}}{\lambda+1}\right) e^{-\lambda x_{i}{ }^{\beta}}\right)^{-1}\left(1-\theta\left(1-\left(1+\frac{\lambda x_{i}^{\beta}}{\lambda+1}\right) e^{-\lambda x_{i}^{\beta}}\right)^{\alpha}\right)^{-1}, \\
& \frac{\partial L}{\partial \lambda}=2 \frac{n}{\lambda}-\frac{n}{\lambda+1}-\sum_{i=1}^{n} x_{i}^{\beta} \\
& +(\alpha-1) \sum_{i=1}^{n}\left(-\left(\frac{x_{i}{ }^{\beta}}{\lambda+1}-\frac{\lambda x_{i}{ }^{\beta}}{(\lambda+1)^{2}}\right) e^{-\lambda x_{i}{ }^{\beta}}+\left(1+\frac{\lambda x_{i}{ }^{\beta}}{\lambda+1}\right) e^{-\lambda x_{i}{ }^{\beta}} x_{i}{ }^{\beta}\right) \\
& \cdot\left(1-\left(1+\frac{\lambda x_{i}{ }^{\beta}}{\lambda+1}\right) e^{-\lambda x_{i}{ }^{\beta}}\right)^{-1}-2 \sum_{i=1}^{n}-\alpha \theta\left(1-\left(1+\frac{\lambda x_{i}{ }^{\beta}}{\lambda+1}\right) e^{-\lambda x_{i}{ }^{\beta}}\right)^{\alpha} \\
& \cdot\left(-\left(\frac{x_{i}{ }^{\beta}}{\lambda+1}-\frac{\lambda x_{i}{ }^{\beta}}{(\lambda+1)^{2}}\right) e^{-\lambda x_{i}{ }^{\beta}}+\left(1+\frac{\lambda x_{i}{ }^{\beta}}{\lambda+1}\right) e^{-\lambda x_{i}{ }^{\beta}} x_{i}{ }^{\beta}\right) \\
& \cdot\left(1-\left(1+\frac{\lambda x_{i}{ }^{\beta}}{\lambda+1}\right) e^{-\lambda x_{i}{ }^{\beta}}\right)^{-1}\left(1-\theta\left(1-\left(1+\frac{\lambda x_{i}^{\beta}}{\lambda+1}\right) e^{-\lambda x_{i}{ }^{\beta}}\right)^{\alpha}\right)^{-1} \text {, } \\
& \frac{\partial L}{\partial \theta}=-\frac{n}{1-\theta}+2 \sum_{i=1}^{n}\left(1-\left(1+\frac{\lambda x_{i}^{\beta}}{\lambda+1}\right) e^{-\lambda x_{i}{ }^{\beta}}\right)^{\alpha}\left(1-\theta\left(1-\left(1+\frac{\lambda x_{i}^{\beta}}{\lambda+1}\right) e^{-\lambda x_{i}{ }^{\beta}}\right)^{\alpha}\right)^{-1} .
\end{aligned}
$$

To find out the MLEs of $(\alpha, \beta, \lambda, \theta)$, say $(\widehat{\alpha}, \widehat{\beta}, \widehat{\lambda}, \widehat{\theta})$ we have to solve the above system of nonlinear equations. As it seems, this system has no closed form solution. We used the NewtonRaphson method to obtain the solution. 
The approximate confidence intervals for the parameters can be based on the asymptotic distribution of the MLEs of $\alpha, \beta, \lambda, \theta$. Under certain regularity conditions, the limiting distribution of $\sqrt{n}(\widehat{\alpha}-\alpha, \widehat{\beta}-\beta, \widehat{\lambda}-\lambda, \widehat{\theta}-\theta)$ as $n \rightarrow \infty$ is a four dimensional normal distribution with zero means and covariance matrix $[E(\mathbf{I})]^{-1}$, where the elements of $\mathbf{I}$ are given in Appendix 7. In practice, $n$ is finite. It is customary that the distribution of $\sqrt{n}(\widehat{\alpha}-\alpha, \widehat{\beta}-\beta, \widehat{\lambda}-\lambda, \widehat{\theta}-\theta)$ is approximated by a four dimensional normal distribution with zero means and covariance matrix $\widehat{\mathbf{I}}^{-1}$, where $\widehat{\mathbf{I}}$ is the $\mathbf{I}$ with $(\alpha, \beta, \lambda, \theta)$ replaced by $(\widehat{\alpha}, \widehat{\beta}, \widehat{\lambda}, \widehat{\theta})$. Let $\widehat{\mathbf{\Sigma}}=\widehat{\mathbf{I}}^{-1}$. Using the approximation, $100(1-\delta)$ percent confidence intervals for $\alpha, \beta, \lambda, \theta$ can be determined as

$$
\widehat{\alpha} \pm z_{\frac{\delta}{2}} \sqrt{\widehat{\Sigma}_{11}}, \quad \widehat{\beta} \pm z_{\frac{\delta}{2}} \sqrt{\widehat{\Sigma}_{22}}, \quad \widehat{\lambda} \pm z_{\frac{\delta}{2}} \sqrt{\widehat{\Sigma}_{33}}, \quad \widehat{\theta} \pm z_{\frac{\delta}{2}} \sqrt{\widehat{\Sigma}_{44}}
$$

respectively, where $z_{\delta}$ is the upper $\delta$ th percentile of the standard normal distribution.

\section{Simulation study}

Here, we used a simulation study to investigate the performance of the accuracy of point estimates of the parameters of the EPLG $(\alpha, \beta, \lambda, \theta)$ distribution. The following steps were followed:

1. Specify the sample size $n$ and the values of the parameters $\alpha, \beta, \lambda$ and $\theta$;

2. Generate $U_{i} \sim$ Uniform $(0,1), i=1, \ldots, n$;

3. Set

$$
X_{i}=\left[-1-\frac{1}{\lambda}-\frac{1}{\lambda} W\left(-(1+\lambda) e^{-1-\lambda},\left(1-\left(\frac{U_{i}}{1-\theta+U_{i} \theta}\right)^{\frac{1}{\alpha}}\right)\right)\right]^{\frac{1}{\beta}}
$$

4. Calculate the MLEs of the four parameters;

5. Repeat steps 2-3, $N$ times;

6. Calculate the mean squared error (MSE) for each parameter.

The comparison is based on MSEs. The MSEs were computed by generating one thousand replications of samples of size $n=10,20, \ldots, 100$ from the EPLG distribution with $(\alpha, \beta, \lambda, \theta)=$ $(1,1.5,2,0.7)$. Figures 4 plot the MSEs of the MLEs of the four parameters. The assessment based on simulation study is that the MSEs for each parameter decreases with increasing sample size.

Figure 4: Plots of the $M S E(\widehat{\alpha}), M S E(\widehat{\beta}), M S E(\widehat{\lambda})$ and $M S E(\widehat{\theta})$ for $(\alpha, \beta, \lambda, \theta)=(1,1.5,2,0.7)$ based on 1000 replications.

\section{Real data applications}

To show the superiority of the EPLG distribution, we compare the results of fitting the EPLG distribution to some other distributions using two real data sets. The first data set represents the strength of $1.5 \mathrm{~cm}$ glass fibres, measured at National physical laboratory, England (see Smith and Naylor (1987)). The second data represents the survival times (in days) of 72 guinea pigs infected 
with virulent tubercle bacilli, observed and reported by Bjerkedal (1960). We compare the fit of the EPLG distribution with the following distributions:

- The generalized Lindley (GL) distribution, introduced by Zakerzadeh and Dolati (2009), with pdf

$$
f_{G L}(x)=\frac{\lambda^{2}(\lambda x)^{\alpha-1}(\alpha+\gamma x)}{(\gamma+\lambda) \Gamma(\alpha+1)} e^{-\lambda x}, x, \theta, \alpha, \gamma>0
$$

- The two-parameter weighted Lindley (WL) distribution, introduced by Ghitany et al. (2011), with pdf

$$
f_{W L}(x)=\frac{\lambda^{c+1}}{(\lambda+c) \Gamma(c)} x^{c-1}(1+x) e^{-\lambda x}, x, \alpha, \lambda>0 .
$$

- The Lindley-Poisson (LP) distribution, introduced by Gui et al. (2014), with pdf

$$
f_{L P}(x)=\frac{\lambda^{2} \theta(x+1) e^{\frac{\theta e^{-\lambda x}(\lambda+\lambda x+1)}{\lambda+1}-\lambda x}}{(\lambda+1)\left(e^{\theta}-1\right)}, x, \theta, \lambda>0 .
$$

- The beta-generalized Lindley (BGL) distribution, introduced by Oluyede and Yang (2014), with pdf

$$
\begin{aligned}
f_{B G L}(x)= & \frac{\alpha \lambda^{2}}{\beta(a, b)(1+\lambda)}(1+x) e^{-\lambda x}\left[1-\left(1+\frac{\lambda x}{\lambda+1}\right) e^{-\lambda x}\right]^{a \alpha-1} \\
& \cdot\left\{1-\left[1-\left(1+\frac{\lambda x}{\lambda+1}\right) e^{-\lambda x}\right]^{\alpha}\right\}^{b-1}, x, a, b, \alpha, \beta, \lambda>0 .
\end{aligned}
$$

- The beta-exponentiated power Lindley (BEPL) distribution, introduced by Pararai et al. (2015), with pdf

$$
\begin{aligned}
f_{B E P L}(x)= & \frac{\alpha \beta^{2} w}{\beta(a, b)(1+\beta)}\left(1+x^{\alpha}\right) x^{\alpha-1} e^{-\beta x^{\alpha}}\left[1-\left(1+\frac{\beta x^{\alpha}}{\beta+1}\right) e^{-\beta x^{\alpha}}\right]^{a w-1} \\
& \cdot\left\{1-\left[1-\left(1+\frac{\beta x^{\alpha}}{\beta+1}\right) e^{-\beta x^{\alpha}}\right]^{w}\right\}^{b-1}, x, a, b, \alpha, \beta, w>0 .
\end{aligned}
$$

- The generalized linear failure rate-geometric (GLFRG) distribution, introduced by Nadarajah et al. (2012), with pdf

$$
f_{G L F R G}(x)=\frac{\alpha(a+b x)(1-\theta) e^{-a x-1 / 2 b x^{2}}\left(1-e^{-a x-1 / 2 b x^{2}}\right)^{\alpha-1}}{\left(1-\theta\left(1-\left(1-e^{-a x-1 / 2 b x^{2}}\right)^{\alpha}\right)\right)^{2}}, x, \alpha, \theta, a, b>0 .
$$

- The McLomax distribution, introduced by Lemonte and Cordeiro (2013), with pdf

$$
f_{\text {McLomax }}(x)=\frac{c \alpha \beta^{\alpha}(\beta+x)^{-(\alpha+1)}}{B\left(a c^{-1}, \eta+1\right)}\left\{1-\left(\frac{\beta}{\beta+x}\right)^{\alpha}\right\}^{a-1}\left[1-\left\{1-\left(\frac{\beta}{\beta+x}\right)^{\alpha}\right\}^{c}\right]^{\eta},
$$

where $x, \alpha, \beta, a, c>0$ and $0 \leq \eta$. 
- The Weibull (W) distribution, introduced by Weibull (1951), with pdf

$$
f_{W}(x)=\alpha \gamma x^{\gamma-1} e^{-\alpha x^{\gamma}}, x, \alpha, \gamma>0 .
$$

- The exponentiated Weibull (EW) distribution, introduced by Mudholkar and Srivastava (1993, 1995), with pdf

$$
f_{E W}(x)=\alpha \gamma \beta^{\gamma} x^{\gamma-1} e^{-(\beta x)^{\gamma}}\left(1-e^{-(\beta x)^{\gamma}}\right)^{\alpha-1}, x, \alpha, \beta, \gamma>0 .
$$

- The modified Weibull (MW) distribution, introduced by Mudholkar and Srivastava (1996), with pdf

$$
f_{M W}(x)=\alpha x^{\gamma-1}(\gamma+\lambda x) e^{\lambda x} e^{-\alpha x^{\gamma} e^{\lambda x}}, x, \alpha, \beta, \gamma>0
$$

Estimates of the parameters of the distributions, Akaike Information Criterion (AIC $=2 p-$ $2 \ln (L))$, Bayesian Information Criterion $(\mathrm{BIC}=p \ln (n)-2 \ln (L))$ and $-2 \ln (L)$ are given in Table

2 for the strength of glass fibres data and in Table 4 for guinea pigs data, where $L=L(\widehat{\boldsymbol{\Theta}})$ is the value of the likelihood function evaluated at the parameter estimates, $n$ is the number of observations, and $p$ is the number of estimated parameters. For more discussion, we present the values of Kolmogorov-Smirnov (KS) statistic, Anderson-Darling statistic (AD) and Cramér-von Mises statistic (CM) for these data sets in Table 4. The smaller the values of these statistics the better the fit. For more details of these statistics, see Chen and Balakrishnan (1995). We also computed the maximum values of the unrestricted and restricted log-likelihoods to obtain the likelihood ratio (LR) statistics for testing some particular cases of the EPLG distribution in Tables 3 and 5. In addition, the empirical scaled TTT transform (Aarset, 1987) and Kaplan-Meier curve can be used to identify the shape of the hazard rate function.

\subsection{Data set 1: Strength of glass fibres data}

The data are: $0.55,0.93,1.25,1.36,1.49,1.52,1.58,1.61,1.64,1.68,1.73,1.81,2.00,0.74,1.04$, $1.27,1.39,1.49,1.53,1.59,1.61,1.66,1.68,1.76,1.82,2.01,0.77,1.11,1.28,1.42,1.50,1.54,1.60$, $1.62,1.66,1.69,1.76,1.84,2.24,0.81,1.13,1.29,1.48,1.50,1.55,1.61,1.62,1.66,1.70,1.77,1.84$, $0.84,1.24,1.30,1.48,1.51,1.55,1.61,1.63,1.67,1.70,1.78,1.89$.

According to the statistics in Table 2, the EPLG distribution fits better than the others for the strength of glass fibres data. Also by using the likelihood ratio $(L R)$ test in Table 3, we test: $H_{0}$ : W distribution versus the alternative hypothesis $H_{1}$ : EPLG distribution. The value of the $L R$ test statistic and the corresponding $p$-value are 6.53858 and 0.04 , respectively. Therefore, the null hypothesis (W distribution) is rejected in favor of the alternative hypothesis (EPLG distribution) at a significance level $>0.04$. Similarly, we can test the other nested distributions versus the EPLG distribution based on Table 3. We see that the EPLG distribution is better than others in that it has the smallest AIC, smallest BIC, smallest AD statistic, smallest CM statistic and smallest K-S statistic. In addition, Figures 5 and 6 show the estimated survival function plot, TTT plot and Kaplan-Meier curve of the fitted distributions for the strength of glass fibres data. These figures show that the EPLG distribution fits better than the other distributions. Consequently, from the values of the statistics in Tables 2, 3 and from Figures 5 and 6, we conclude that the EPLG distribution gives the better fit for strength of glass fibres data. 
Table 2: Estimates of fitted distributions for strength of glass fibres data.

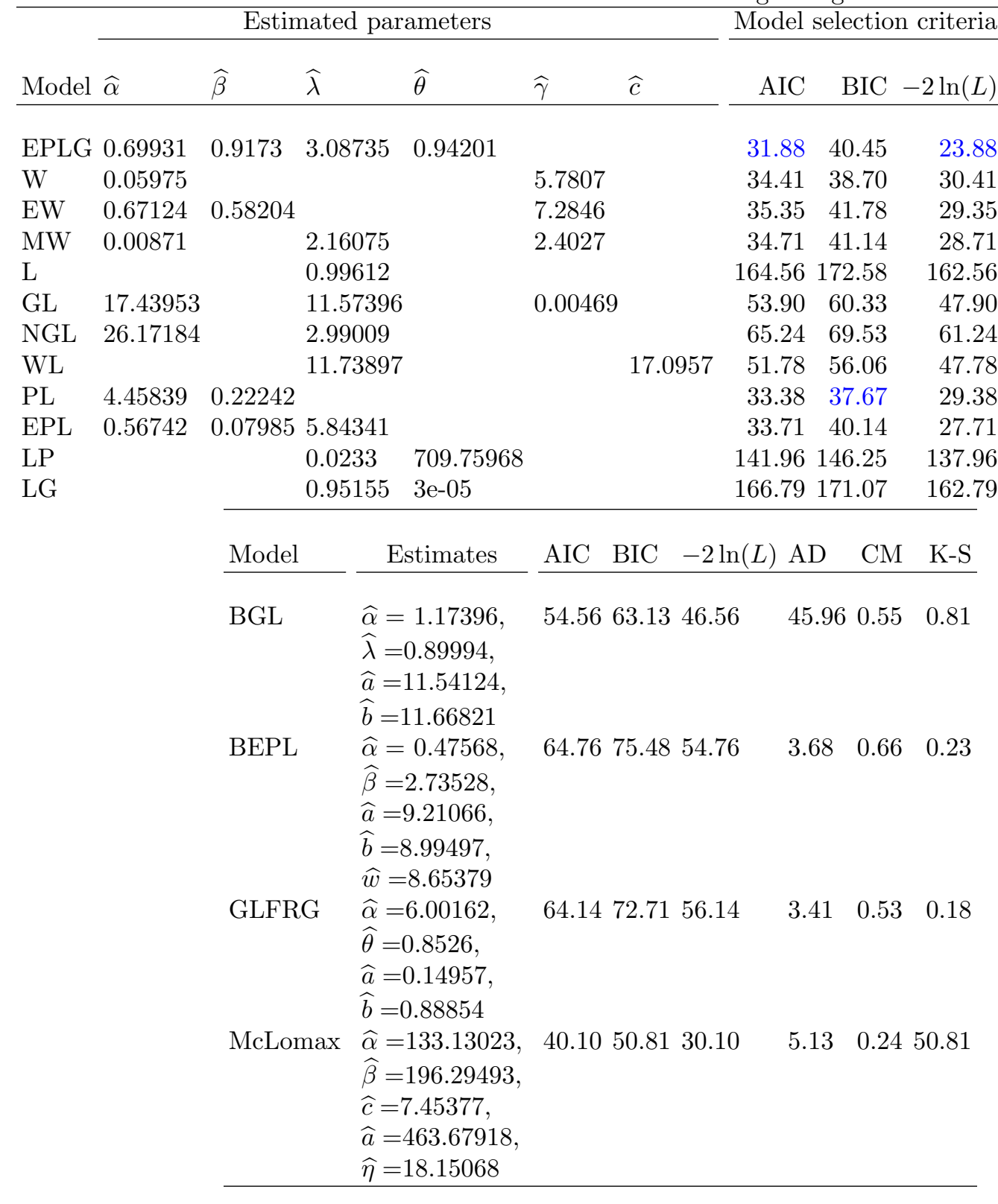

Figure 5: Estimated survival function and the empirical survival for strength of glass fibres data.

Figure 6: Empirical TTT-plot (top left), estimated hazard rate function (top right), estimated survival function (bottom) for the fitted distributions to the strength of glass fibres data. 
Table 3: LR statistics and corresponding $p$-values for strength of glass fibres data.

\begin{tabular}{|c|c|c|c|c|c|c|c|c|c|c|c|}
\hline & $\mathrm{W}$ & EW & & GL & NGL & WL & $\begin{array}{l}\text { PL EPL } \\
\end{array}$ & LP & $\overline{\mathrm{LG}}$ & BGL & GLF \\
\hline & EPLG & EPLG & EPLG & EPLG & EPLG & EPLG & & & & LG & \\
\hline & 6.54 & 5.48 & & & 37.36 & & & & 138.91 & 22.68 & \\
\hline & 0.04 & 0.02 & 00 & 0.0 & 0.00 & 0.0 & C & 0.00 & 0.00 & 0.00 & .00 \\
\hline
\end{tabular}

\subsection{Data set 2: Guinea pigs data}

The data are: $10,33,44,56,59,72,74,77,92,93,96,100,100,102,105,107,107,108,108,108$, $109,112,113,115,116,120,121,122,122,124,130,134,136,139,144,146,153,159,160,163$, $163,168,171,172,176,183,195,196,197,202,213,215,216,222,230,231,240,245,251,253$, $254,254,278,293,327,342,347,361,402,432,458,555$.

Figure 7: Estimated survival function and the empirical survival function for the guinea pigs data.

Figure 8: Empirical TTT-plot (top left), estimated hazard rate function (top right), estimated survival function (bottom) for the fitted distributions for the guinea pigs data.

According to the statistics in Table 4, the EPLG distribution fits better than the others for the guinea pigs data. Also by using the likelihood ratio $(L R)$ test in Table 5 , we test: $H_{0}$ : W distribution versus the alternative hypothesis $H_{1}$ : EPLG distribution. The value of the $L R$ test statistic and the corresponding $p$-value are 5.48 and 0.06 , respectively. Therefore, the null hypothesis (W distribution) is rejected in favor of the alternative hypothesis (EPLG distribution) at a significance level $>0.06$. Similarly, we can test the other nested distributions versus the EPLG distribution based on Table 5. We see again that the EPLG distribution is better than others in that it has the smallest AIC, smallest BIC, smallest AD statistic, smallest CM statistic and smallest K-S statistic. In addition, Figures 7 and 8 show the estimated survival function plot, TTT-plot and KaplanMeier curve of the fitted distributions to the guinea pigs data. These figures show that the EPLG distribution fits better than the other distributions. Consequently, from the values of the statistics in Tables 4, 5 and from Figures 7 and 8, we conclude that the EPLG distribution gives the better fit for the guinea pigs data.

In both data applications, some of the fitted distributions (BEPL and McLomax) are not nested with the EPLG distribution and have larger number of parameters. Yet the EPLG distribution provides better fits than these distribution in that it yielded smaller AICs, smaller BICs, smaller AD statistic, smaller CM statistic and smaller K-S statistic.

The parameter estimates of the best fitting EPLG distribution to the glass fiber data can be interpreted as follows. Suppose each glass fiber is made of up a geometric number of components working in parallel and that the failure time of each component is EPL distributed, so the glass fiber will break if and only if all its components fail. According to the parameter estimates, there is an average of 1.06 components working in parallel with a variance of 0.07 . The mean failure time of each component is 0.301 . The variance of the failure time of each component is 0.137 .

The parameter estimates of the best fitting EPLG distribution to the guinea pigs data can be interpreted as follows. Suppose each guinea pig is made of up a geometric number of components working in parallel and that the failure time of each component is EPL distributed, so the pig will 
Table 4: Estimates of fitted distributions for guinea pigs data.

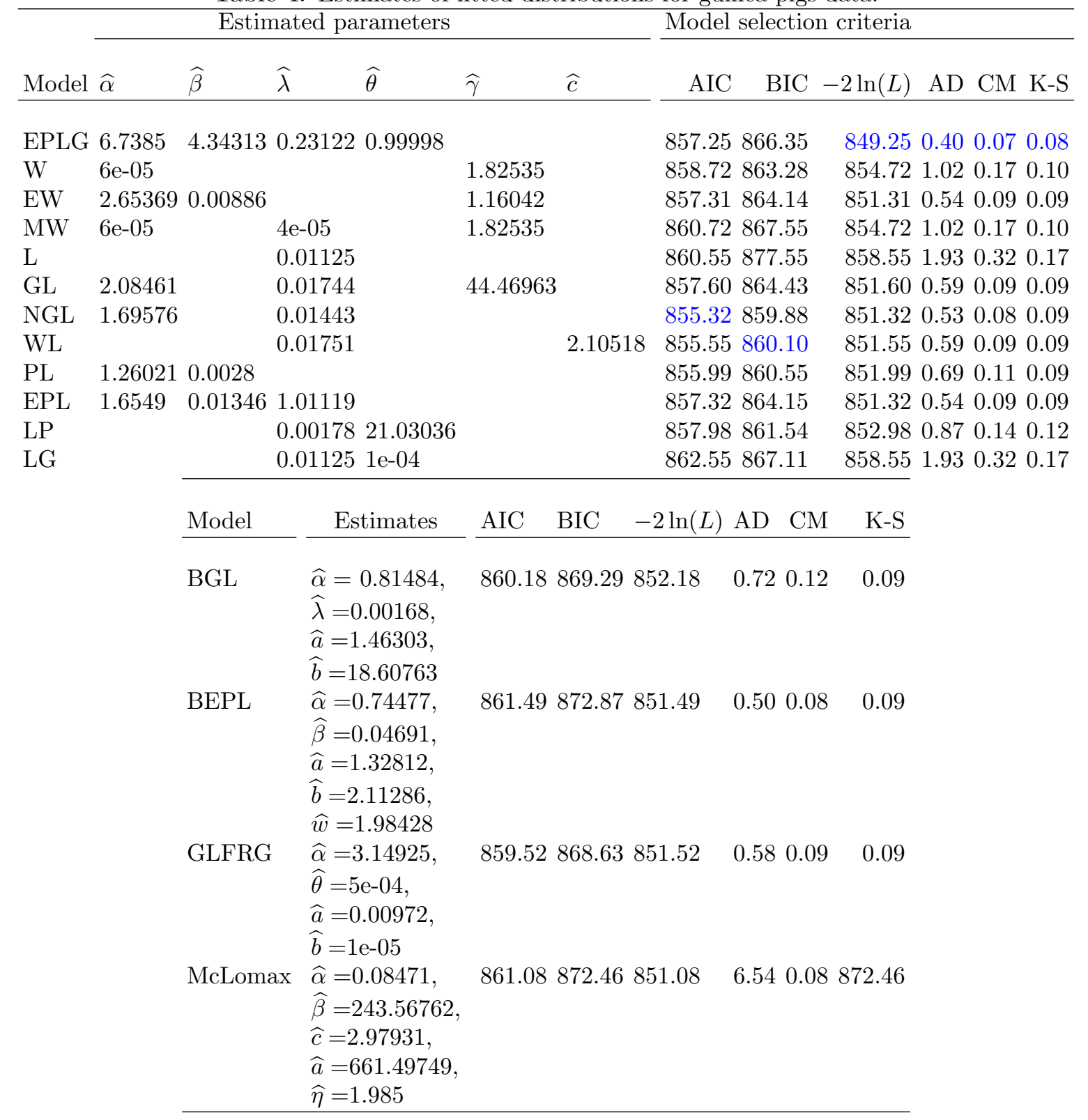


Table 5: LR statistics and corresponding $p$-values for guinea pigs data.

\begin{tabular}{|c|c|c|c|c|}
\hline$H_{0}$ & W & $\mathrm{L}$ & LG BGL & GLFRG \\
\hline$H_{1}$ & EPLG & EPLG & EPLG EPLG & EPLG \\
\hline LR statistics & 5.48 & 9.31 & $9.31 \quad 2.94$ & 2.27 \\
\hline$p$-value & 0.06 & 0.03 & $\begin{array}{lll}0.01 & 0.00\end{array}$ & 0.00 \\
\hline
\end{tabular}

die if and only if all its components fail. According to the parameter estimates, there is an average of 1.00 component working in parallel with a variance of $2 \times 10^{-5}$. The mean failure time of each component is 1.90. The variance of the failure time of each component is 0.026 .

\section{Conclusions}

We introduced a four-parameter lifetime distribution called the exponentiated power Lindley geometric (EPLG) distribution, a mixed distribution of the exponentiated power Lindley and geometric distributions. The EPLG distribution is flexible in modelling various types of failure data with possibly bathtub-shaped hazard rate functions. The EPLG distribution is more flexible than the Weibull, exponentiated Weibull, modified Weibull, Lindley, generalized Lindley, new generalized Lindley, weighted Lindley, power Lindley, exponentiated power Lindley, Lindley Poisson, Lindley geometric, beta generalized Lindley, beta exponentiated power Lindley, generalized linear failure rate geometric and McLomax distributions. The mathematical properties of the EPLG distribution derived include explicit expressions for the density function of the order statistics, their moments, quantiles, moments, residual life moments and probability weighted moments. The simulation study and two real data applications discussed show the potential of the EPLG distribution. We hope that the EPLG distribution may attract wider applications in survival analysis.

\section{Acknowledgments}

The authors would like to thank the Editor and the two referees for careful reading and comments which greatly improved the paper.

\section{References}

[1] Aarset, M.V. How to identify bathtub hazard rate. IEEE Transactions on Reliability, 36 (1987) 106-108.

[2] Adamidis, K. and Loukas, S. A lifetime distribution with decreasing failure rate. Statistics and Probability Letters, 39 (1998) 35-42.

[3] Asgharzadeh, A., Bakouch S.H., Nadarajah, S. and Esmaeili, L. A new family of compound lifetime distributions. Kybernetika, 50 (2014) 142169.

[4] Ashour, S.K. and Eltehiwy, M.A. Exponentiated power Lindley distribution. Journal of Advanced Research, (2014), http://dx.doi.org/10.1016/j.jare.2014.08.005 
[5] Bagheri, S.F., Samani, E.B. and Ganjali, M. The generalized modified Weibull power series distribution: Theory and applications. Computational Statistics and Data Analysis, 94 (2016) 136-160.

[6] Bakouch, H.S., Al-Zahrani, B.M., Al-Shomrani, A.A., Marchi, V.A.A. and Louzada, F. An extended Lindley distribution. Journal of the Korean Statistical Society, 41 (2012) 75-85.

[7] Barreto-Souza, W. and Cribari-Neto, F. A generalization of the exponential-Poisson distribution. Statistics and Probability Letters, 79 (2009) 2493-2500.

[8] Barreto-Souza, W., Morais, A.L. and Cordeiro, G.M. The Weibull-geometric distribution. Journal of Statistical Computation and Simulation, 81 (2011) 645-657.

[9] Chahkandi, M. and Ganjali, M. On some lifetime distributions with decreasing failure rate. Computational Statistics and Data Analysis, 53 (2009) 4433-4440.

[10] Chen, G. and, Balakrishnan, N. A general purpose approximate goodness-of-fit test. Journal of Quality Technology, 27 (1995) 154-161.

[11] Corless, R.M., Gonnet, G.H., Hare, D.E.G., Jeffrey, D.J. and Knuth, D.J. On the Lambert W function. Advances in Computational Mathematics, 5 (1996) 329-359.

[12] Ghitany, M.E. On a recent generalization of gamma distribution. Communications in Statistics: Theory and Methods, 27 (1998) 223-233.

[13] Ghitany, M.E., Al-Mutairi, D.K., Balakrishnan, N. and Al-Enezi L.J. Power Lindley distribution and associated inference. Computational Statistics and Data Analysis, 64 (2013) $20-33$.

[14] Ghitany, M.E., Al-Qallaf, F., Al-Mutairi, D.K. and Hussain, H.A. A two-parameter weighted Lindley distribution and its applications to survival data. Mathematics and Computers in Simulation, 81 (2011) 1190-1201.

[15] Ghitany, M.E., Atieh, B. and Nadarajah, S. Lindley distribution and its application. Mathematics and Computers in Simulation, 78 (2008) 493-506.

[16] Giorgi, G.M. Concentration index, Bonferroni. Encyclopedia of Statistical Sciences, volume 2. Wiley, New York (1998) 141-146.

[17] Giorgi, G.M. and Crescenzi, M. A look at the Bonferroni inequality measure in a reliability framework. Statistica, 4 (2001) 571-583.

[18] Greenwood, J.A., Landwehr, J.M., Matalas, N.C. and Wallis, J.R. Probability weighted moments: Definition and relation to parameters of several distribution expressible in inverse form. Water Resources Research, 15 (1979) 1049-1054.

[19] Gui, W. Zhang, S. and Lu, X. The Lindley-Poisson distribution in lifetime analysis and its properties. Journal of Mathematics and Statistics, (2014) 10-63.

[20] Gupta, P.L. and Gupta, R.C. On the moments of residual life in reliability and some characterization results. Communications in Statistics: Theory and Methods, 12 (1983) 449-461.

[21] Johnson, N.L., Kotz, S. and Balakrishnan, N. Continuous Univariate Distributions, volume 1, second edition. New York, Wiley (1994). 
[22] Kundu, C. and Nanda, A.K. Some reliability properties of the inactivity time. Communications in Statistics: Theory and Methods, 39 (2010) 899-911.

[23] Hosking, J.R.M. The theory of probability weighted moments. IBM Research Report, RC 12210, Yorktown Heights, New York (1986).

[24] Hosking, J.R.M., Wallis, J.R. and Wood, E.F. Estimation of the generalized extreme value distribution by the method of probability weighted moments. Technometrics, 27 (1985) 251261.

[25] Lai, C.D., Xie, M. and Murthy, D.N.P. A modified Weibull distribution. IEEE Transactions on Reliability, 52 (2003) 33-37.

[26] Lemonte, A.J. and Cordeiro, G.M. An extended Lomax distribution. Statistics, 47 (2013) 800-816.

[27] Lindley, D.V. Fiducial distributions and Bayes' theorem. Journal of the Royal Statistical Society, Series B, 20 (1958) 102-107.

[28] Louzada-Neto, F., Roman, M. and Cancho, V.G. The complementary exponential-geometric distribution for lifetime data. Computational Statistics and Data Analysis, 55 (2011) 25162524 .

[29] Mahmoudi, E. and Jafari, A.A. Generalized exponential-power series distributions. Computational Statistics and Data Analysis, 56 (2012) 4047-4066.

[30] Mahmoudi, E. and Jafari, A.A. The compound class of linear failure rate-power series distributions: Model, properties and applications. arXiv: 1402.5282 (2014).

[31] Mahmoudi, E. and Sepahdar, A. Exponentiated Weibull-Poisson distribution: Model, properties and applications. Mathematics and Computers in Simulation, 92 (2013) 76-97.

[32] Mahmoudi, E., Sepahdar, A. and Lemonte, A. Exponentiated Weibull-logarithmic distribution: Model, properties and applications. arXiv:1402.5264 (2014).

[33] Mahmoudi, E. and Shiran, M. Exponentiated Weibull power series distributions and its applications. arXiv:1206.4008, arXiv:1204.4248, arXiv:1212.5586.

[34] Merovci. F. and Elbatal, I. Transmuted Lindley-geometric and its application. Journal of Statistics Applications and Probability, 3 (2014) 77-91.

[35] Mi, J. Bathtub failure rate and upside-down bathtub mean residual life. IEEE Transactions on Reliability, 44 (1995) 388391.

[36] Moors, J.J.A. A quantile alternative for kurtosis. The Statistician, 37 (1988) 25-32.

[37] Morais, A.L. and Barreto-Souza, W. A compound class of Weibull and power series distributions. Computational Statistics and Data Analysis, 55 (2011) 1410-1425.

[38] Mudholkar, G.S. and Srivastava, D.K. Exponentiated Weibull family for analyzing bathtub failure-real data. IEEE Transactions on Reliability, 42 (1993) 299-302.

[39] Mudholkar, G.S., Srivastava, D.K. and Friemer, M. The exponentiated Weibull family: A reanalysis of the bus-motor-failure data. Technometrics, 37 (1995) 436-445. 
[40] Mudholkar, G.S., Srivastava, D.K. and Kollia, G.D. A generalization of the Weibull distribution with application to the analysis of survival data. Journal of the American Statistical Association, 91 (1996) 1575-1583.

[41] Nadarajah, S., Bakouch, H.S. and Tahmasbi, R.A. Generalized Lindley distribution. Sankhya $B, 73$ (2011) 331-359.

[42] Nadarajah, S., Shahsanaei, F. and Rezaei, S. A new four-parameter lifetime distribution. Journal of Statistical Computation and Simulation, 84 (2014) 248-263.

[43] Nanda, A.K., Singh, H., Misra, N. and Paul, P. Reliability properties of reversed residual lifetime. Communications in Statistics: Theory and Methods, 32 (2003) 20312042.

[44] Oluyede, B. and Yang T. A new class of generalized Lindley distributions with applications. Journal of Statistical Computation and Simulation, http://dx.doi.org/10.1080/00949655.2014.917308

[45] Pararai, M., Warahena-Liyanage, G. and Oluyede, B.O. A new class of generalized power Lindley distribution with applications to lifetime data. Theoretical Mathematics and Applications, 5 (2015) 53-96.

[46] Park, K.S. Effect of burn-in on mean residual life. IEEE Transactions on Reliability, 34 (1985) $522-523$.

[47] Ramos, M.W.A., Percontini, A., Cordeiro, G.M. and Ronaldo da Silva, V. The Burr XII negative Binomial distribution with applications to lifetime data. International Journal of Statistics and Probability, 1 (2015) 109-125.

[48] Silva, R.B., Bourguignon, M., Dias, C.R.B. and Cordeiro, G.M. The compound class of extended Weibull power series distributions. Computational Statistics and Data Analysis, $\mathbf{5 8}$ (2013) 352-367.

[49] Smith, R.L. and Naylor, J.C. A comparison of maximum likelihood and Bayesian estimators for the three-parameter Weibull distribution. Applied Statistics, 36 (1987) 358-369.

[50] Tahmasbi, R. and Rezaei, S. A two-parameter lifetime distribution with decreasing failure rate. Computational Statistics and Data Analysis, 52 (2008) 3889-3901.

[51] Tang, L.C., Lu, Y. and Chew, E.P. Mean residual life distributions. IEEE Transactions on Reliability, 48 (1999) 68-73.

[52] Tojeiro, C., Louzada, R. and Borges, P. The complementary Weibull geometric distribution. Journal of Statistical Computation and Simulation, 6 (2014) 1345-1362.

[53] Weibull, W. A statistical distribution function of wide applicability. Journal of Applied Mechanics, Transactions ASME, 18 (1951) 293-297.

[54] Zakerzadeh, H. and Dolati, A. Generalized Lindley distribution. Journal of Mathematical Extension, 3 (2009) 13-25.

[55] Zakerzadeh, H. and Mahmoudi, E. A new two-parameter lifetime distribution: Model and properties. arXiv:1204.4248 (2012). 